von roher und gekochter Milch mittels Benzidins gemacht und darauf hingewiesen, daß sich diese Reaktion auch umgekehrt zur Prüfung auf Wasserstoffsuperoxyd in Milch verwenden läßt. Inzwischen haben wir unsere Reaktion auf ihre Empfindlichkeit geprüft und gefunden, daß bei einem Wasserstoffsuperoxydgehalt von $0,05 \mathrm{mg}$ in $5 \mathrm{cem}$ Milch die Reaktion noch deutlich eintritt, ein geringerer Gehalt sich aber nicht mehr zu erkennen gibt. Weder Nitrate noch Eisenoxydsalze scheinen die Reaktion zu beeinträchtigen.

\title{
Über die Fiehe'sche Reaktion zur Erkennung und Unterscheidung von Kunsthonigen und Naturhonigen.
}

Bezugnehmend auf die kürzlich in der Zeitschrift für öffentliche Chemie (1908, 14, 352) erschienene kurze Mitteilung von Dr. Drawe • Görlitz „Beitrag zur Dr. Fiehe'schen Reaktion auf Invertzucker im Honig" kann ich bestätigen, daß naturreine Honige schon nach halbstündigem Erwärmen im Wasserbade die Fiehe'sche Reaktion sowohl mit 35\%-iger als auch mit 25\%-iger Salzsäure deutlich und bleibend geben. Da ich eine größere Anzahl von Versuchen angestellt habe, behalte ich mir ausführlichere Mitteilungen hierüber vor.

E, v. Raumer.

\section{Reierate. \\ Milch und Käse.}

B. J. Slowzow: Zur Frage über Gerinnung der Milch durch das Labferment. (Russki Wratsch 1906, 5, 1178-1181.) - Die Gerinnung von Milch und Albumosen durch das Labferment wird von den Autoren recht verschieden erklärt. Nach Hammarsten zerfällt das Mileheasein dureh die Einwirkung von Labferment in eine geringe Menge löslichen Eiweißes und in Paracasein, welches in Gegenwart von Calciumsalzen schnell ausfällt. Die Ansicht, daß das Labferment einen Zerfall des Caseins bewirkt, wurde auch von Ringer, Artus, Pages, Courant, Edkins und Laqueur bestätigt. Letzterer fand, daß das Paracasein ein geringeres Molekulargewicht besitzt, als das Casein. Fuld dagegen ist der Ansicht, dass die Umwandlung des Caseins in Paracasein nicht durch den Zerfall des ersteren, sondern durch eine intramolekulare Umsetzung der Teilchen geschieht. Nach Loewenhard hat das Paracasein ein größeres Molekulargewicht, als das Casein, sodaß also das Labferment synthetisch auf Casein wirkt. A. Danilewski fand, dal der Zusatz von Labferment zu Pepton- und Albumosenlösungen einen voluminösen Eiweißniederschlag "Plastein" hervorruft, und faßt den Prozeß der Labwirkung als einen dem hydrolytischen entgegengesetzten - als Albuminisation auf. Nach Sawjalow und Kuraew ist das Plastein von dem Ausgangseiweil verschieden und Salaskin schlägt vor, das Plastein Labalbumose zu nennen und meint, dafo sein Bildungsprozeß der Hydrolyse sehr nahe komme. Daß das Lab- und Pepsinferment ganz verschieden von einander sind, galt für erwiesen, bis M. Nentzki bei der Untersuchung der Fermente des natürlichen Magensaftes fand, daß die sogen. Pepsinkörner stets außer Pepsin auch Lab und Invertin enthalten. Nach Nentzki stellt das Pepsinkorn ein großes Eiweißteilchen mit einer Reihe von Ketten dar, welche in der einen oder anderen Richtung wirken. J. P. Pawlow und Paraschtschuk kamen ebenfalls zu dem Schlusse, daß das Labferment und Pepsin eins ist und von- 